\title{
Prognosis and treatment of polymyositis with particular reference to steroid resistant patients
}

\author{
M YOSHIOKA, T OKUNO, AND H MIKAWA \\ Departments of Paediatrics, Kobe General Hospital and Faculty of Medicine, Kyoto University, Japan
}

SUMMARY Eight boys and six girls with polymyositis examined between 1967 and 1982 were studied. The mean age of disease onset was 5 years 5 months. The initial regimen was prednisolone, 1.2 to $2.3 \mathrm{mg} / \mathrm{kg} / \mathrm{day}$, and after four weeks this dose was decreased gradually to a maintenance level of 5 to $20 \mathrm{mg}$ on alternate days. The total treatment period was 3 years 6 months on average. Eleven of the 14 children had a uniphasic course, and steroids were stopped without a resurgence of the disease: three were refractory to steroid treatment. One of these died of a cardiomyopathy seven years after the onset of the illness despite treatment with steroids and cyclophosphamide; the second was treated with adrenocorticotrophic hormone after prednisolone, but without benefit; and in the third a series of treatment with lympho-plasmapheresis and cyclophosphamide resulted in some improvement.

As cardiac involvement in polymyositis may become overt if the disease persists for many years, patients refractory to steroids should be given alternative treatment.

Polymyositis in childhood is characterised by muscle weakness and is sometimes associated with skin changes (dermatomyositis): this entity is usually classified as a collagen-vascular disease. Although the aetiology of polymyositis remains unknown, there is substantial evidence to suggest the immunologic mechanisms contribute to the muscle and connective tissue injury. ${ }^{12}$ Treatment with prednisolone at several dose levels has been suggested. ${ }^{3-6}$ Cytotoxic chemotherapy is also often prescribed for patients with an unsatisfactory response to prednisolone. $^{7-9}$

Between 1967 and 1982, 14 patients with polymyositis were seen on our clinics. Three of the 14 $(20 \%)$ did not benefit from prednisolone and were thought to be steroid resistant. One of the three died of cardiomyopathy seven years after the onset of the illness and in another, severe contractures occurred and use of a wheelchair became necessary. Although an improved prognosis in a series of patients with polymyositis in childhood treated with steroids has been reported, ${ }^{3-5}$ we found severe residual symptoms in some children with this disease.

\section{Materials and methods}

Between 1967 and 1982, 14 children were studied at the Paediatric Departments of Kobe General and Kyoto University Hospitals. The data obtained are summarised in Tables 1 and 2 . The age of onset ranged between 2 and 14 years; mean age 5 years 5 months. The diagnosis of polymyositis was based on the clinical findings of muscle weakness and skin rash, and confirmation was obtained by either enzyme abnormality, a muscle biopsy showing evidence of inflammatory myositis, or an abnormal electromyogram. The duration of follow up was 3 years 2 months to 11 years 5 months. The initial regimen of prednisolone was 1.2 to $2.3 \mathrm{mg} / \mathrm{kg} /$ day. After four weeks, this dose was reduced, dependent upon improvement in the clinical status and reversal of enzyme abnormality. The dose of the steroid was decreased further to a maintenance level of 5 to 20 $\mathrm{mg}$, on alternate days. On average, the total treatment period was 3 years 6 months. Three of the 14 patients (cases 2, 10, and 13) were resistant to steroid and were given alternative treatment.

The grading system used for disability was the one employed by Rose and Walton. ${ }^{10}$

(1) No abnormality on examination and full functional recovery.

(2) No abnormality on examination, but easily tired with reduced exercise tolerance.

(3) Minimal degree of atrophy or weakness in one 


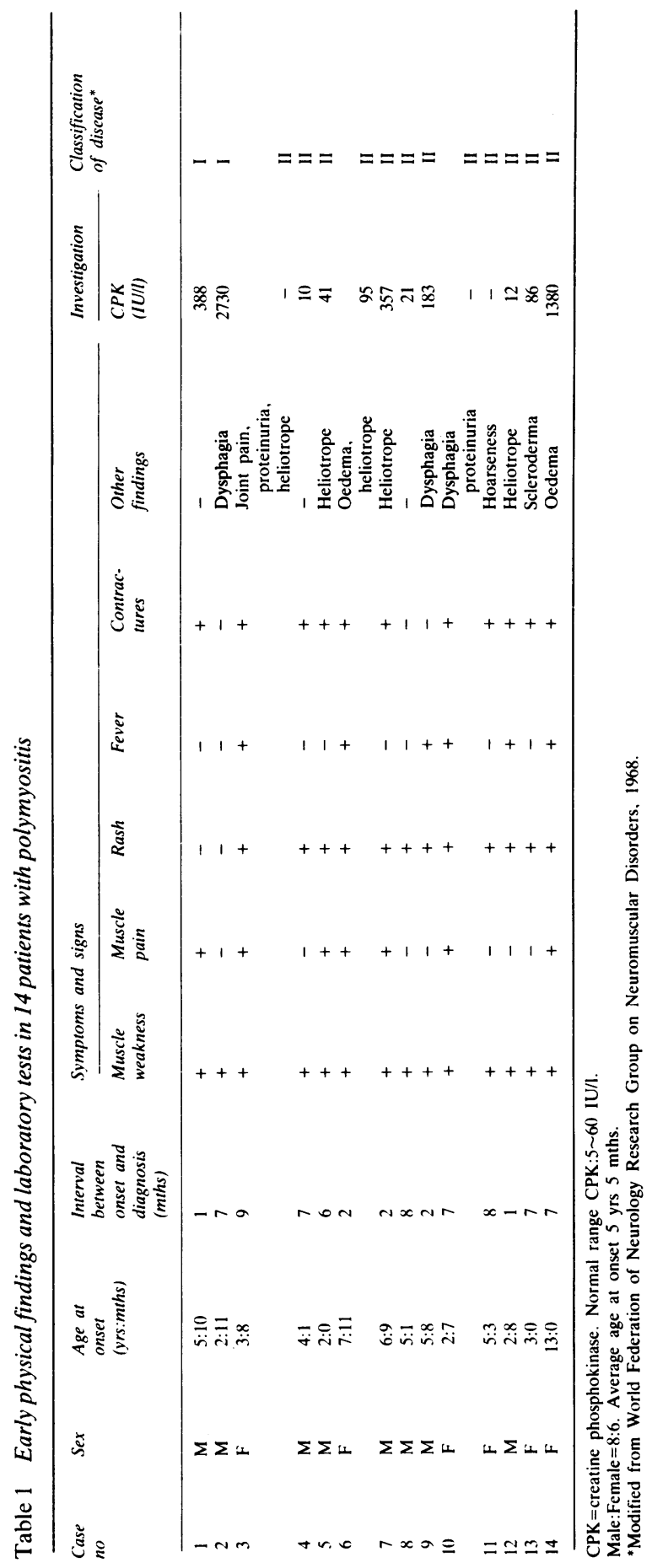




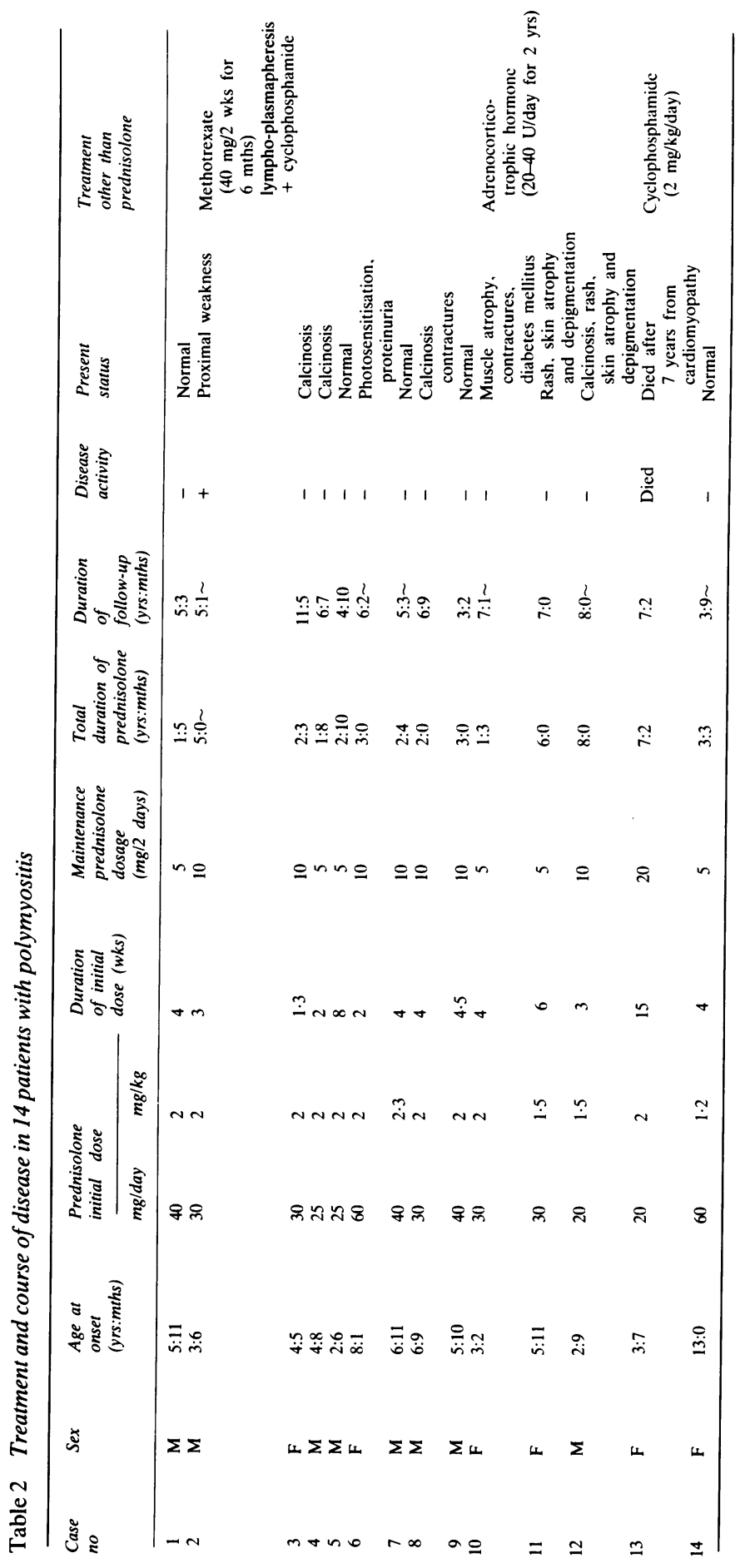


or more muscle groups without functional impairment.

(4) Incomplete recovery, waddling gait, unable to run but able to climb stairs without needing arm support.

(5) Incomplete recovery, noticeable waddling gait and accentuated lumbar lordosis, unable to climb stairs or rise from a standard high chair without needing arm support.

(6) Unable to walk without assistance.

\section{Results}

The essential clinical features and laboratory tests are summarised in Table 1. A skin rash characteristic of dermatomyositis occurred in $12(86 \%)$ of the children. Two of the 12 were classified as dermatomyositis with other connective tissue disease; one (case 13) with systemic sclerosis and the other (case 10) with rheumatoid arthritis. These two children were resistant to steroid treatment. In three patients who complained of difficulty in swallowing (cases 2 ,
9 , and 10), two were resistant to steroid (cases 2 and 10). Rheumatoid factors were positive in four; two of these were resistant to steroid and showed positive reactions during the clinical course, compared with the remaining two in whom the rheumatoid factor was positive only in the earlier, active stage of disease. The electromyographic findings were those of a primary myopathy and were consistently abnormal in all patients. Muscle biopsy was undertaken in all patients. The findings included perivascular and interstitial infiltration of round mononuclear cells and focal muscle degeneration and regeneration. One child had a completely normal muscle biopsy.

Table 2 outlines the treatment and clinical course. Eleven of the 14 patients had an uniphasic course and steroids were stopped without a resurgence of the disease. Three were thought to be resistant to steroid: one died of cardiomyopathy; another has to use a wheelchair; and the third still has active disease and relapses frequently. Two of these three patients resistant to steroid are presented below.

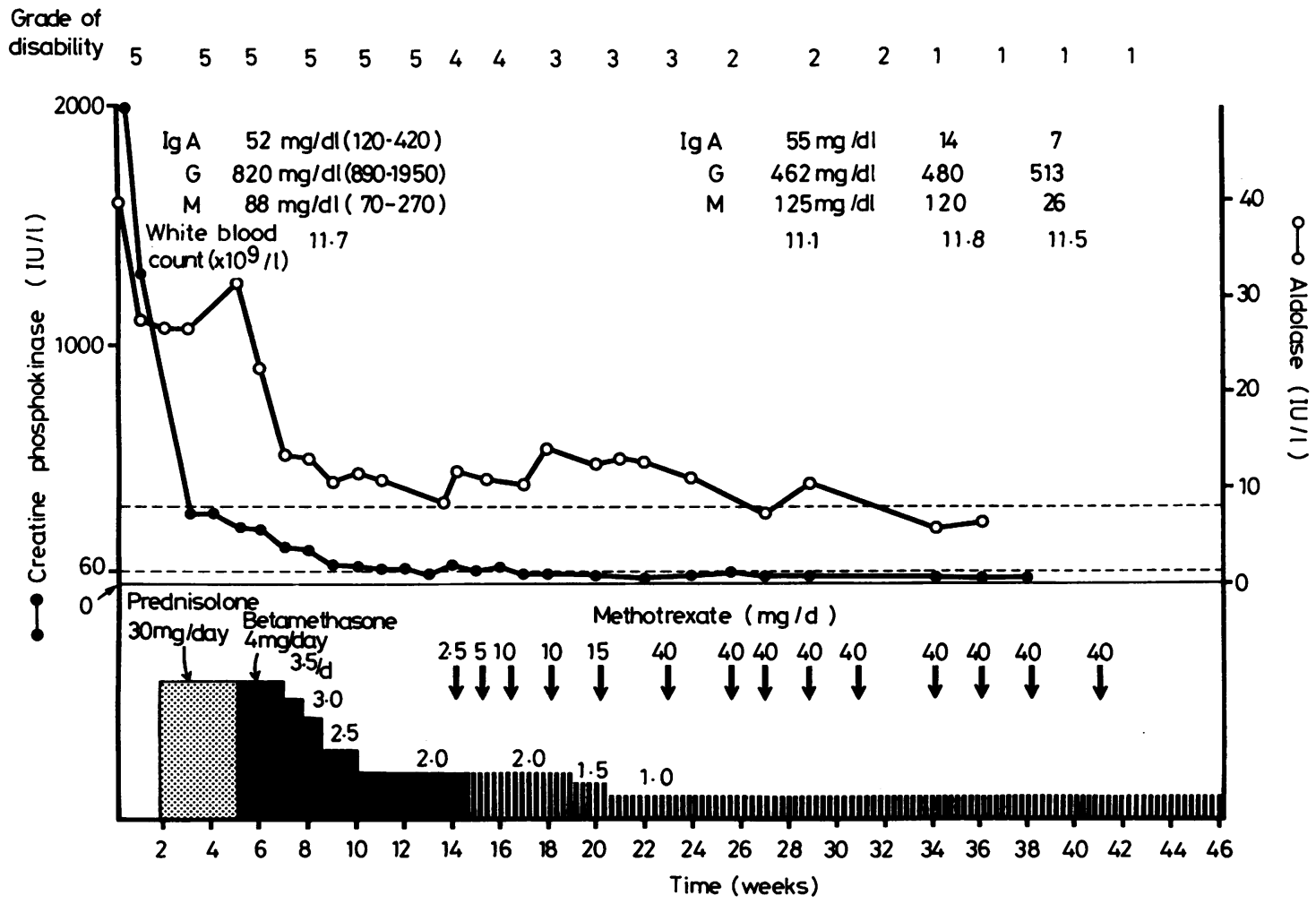

Fig. 1 Early clinical course and treatment of the patient in case 2. 
Case 2. This boy aged 8 years 2 months developed polymyositis when he was 2 years 10 months old. His family history was unremarkable. The illness began with weakness of the legs and arms; facial oedema, erythematous butterfly rash, intermittent fever, and arthralgias were not present. Within three months he could neither climb the stairs nor stand, and he suffered slight dysphagia. The initial creatine phosphokinase activity was $2730 \mathrm{IU} / 1$, lactate dehydrogenase $468 \mathrm{IU} / 1$, serum glutamic oxaloacetic transaminase $110 \mathrm{IU} / \mathrm{l}$, and aldolase 53.0 IU/l. Diagnosis of polymyositis was confirmed by electromyography and muscle biopsy. The patient was treated with $30 \mathrm{mg} /$ day of prednisolone (2 $\mathrm{mg} / \mathrm{kg} /$ day). Despite the noticeable drop in creatine phosphokinase after the initial treatment with prednisolone, muscle weakness continued and aldolase concentrations did not decrease (Fig. 1). The patient was then treated with betamethasone (4 $\mathrm{mg} /$ day) for 14 weeks but without clinical improvement. Methotrexate, $40 \mathrm{mg} / 2$ weeks $(2 \mathrm{mg} / \mathrm{kg} / 2$ weeks) was added and betamethasone, $1.0 \mathrm{mg} / 2$ days, was continued. Considerable improvement was noted over the next four months and the patient's electromyography and aldolase activity became normal.

Over the next three and a half years he had four relapses after an upper respiratory tract infection, varicella, viral meningitis, and viral pneumonia (Fig. 2). His gradual downhill course continued. Muscle strength lessened, muscle tissue became atrophic and creatine phosphokinase activities were around $500 \mathrm{IU} / 1$.

On examination there was moderate proximal extremity weakness and atrophy. Prednisolone treatment was continued and lymphocytapheresis together with plasmapheresis (lympho-plasmapheresis), twice or three times a week, was initiated. Lympho-plasmapheresis was performed using the Hemonetics-PEX (Hemonetics USA), an intermittent flow cell separator. The treatment was given twice or three times a week for 12 weeks followed by twice a month for the next eight months (a total of 34). During each exchange, between 450 and $980 \mathrm{ml}$

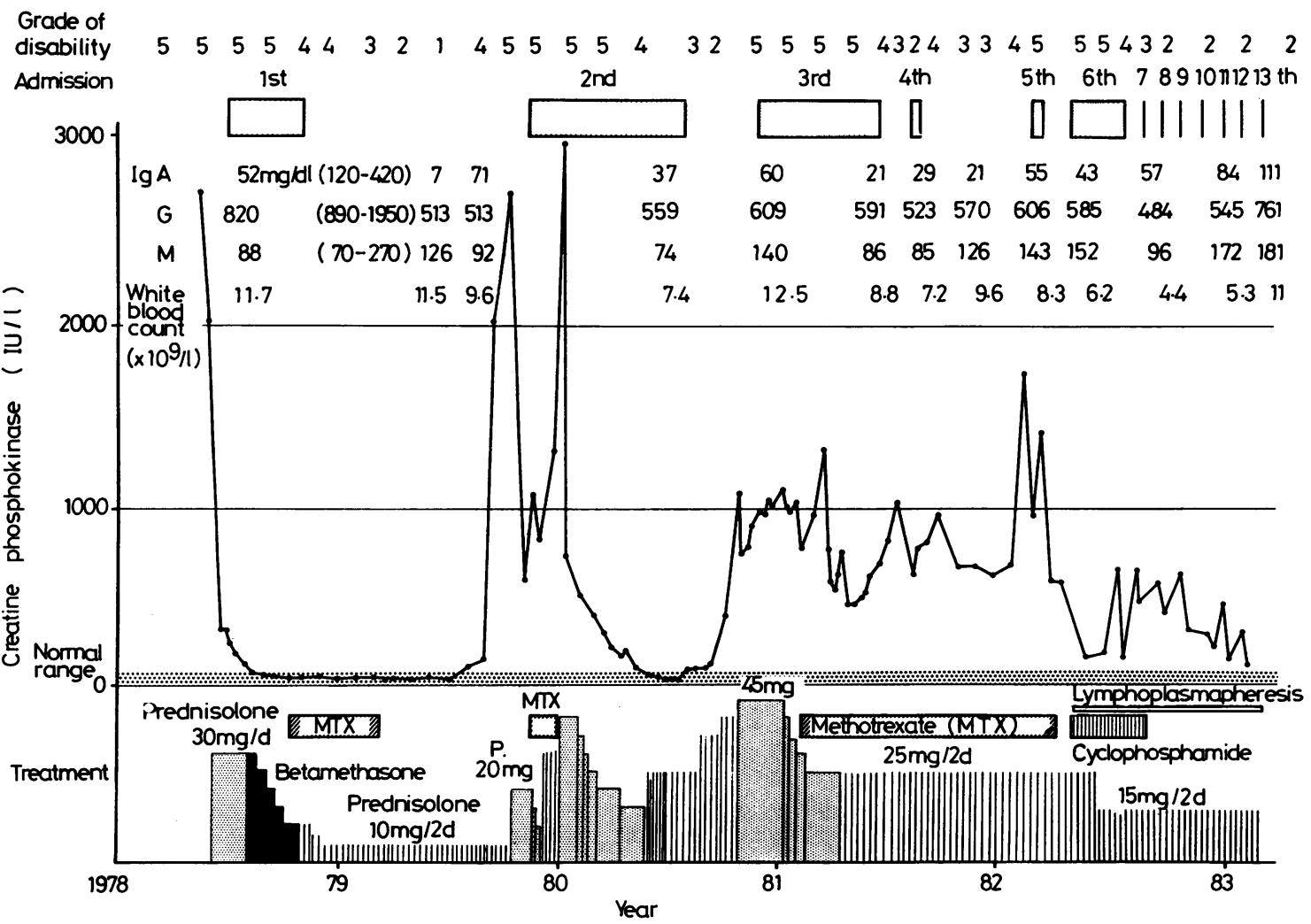

Fig. 2 Entire clinical course and treatment of the patient in case 2. 
of plasma and $8 \times 10^{8}$ to $1.9 \times 10^{9}$ lymphocytes were withdrawn and replaced with plasmanate. Prednisolone, $25 \mathrm{mg}$ every other day, was continued and, as improvement occurred, was reduced to $15 \mathrm{mg}$ every other day. Cyclophosphamide was added after the seventh lympho-plasmapheresis, but was eventually stopped after the 23 rd because of clinical improvement.

Circulating immune complexes were determined with solid phase $\mathrm{Clq}$ binding assays, but only once (after the first lympho-plasmapheresis) was there a slightly raised concentration. By the 10th lymphoplasmapheresis the creatine phosphokinase activity had decreased and the child's strength had steadily improved. After the 16th, muscle testing showed slight residual proximal weakness and the patient could climb the stairs. A reinfection of varicella developed after the 34th treatment with lymphoplasmapheresis, but he made a rapid recovery.

Case 13. This girl aged 10 years and 8 months had begun to experience difficulty in walking 7 years before admission to hospital. At the age of 3 years 8 months there had been severe distal muscle atrophy and depigmentation of skin of the face, arms, and legs. The wrists and ankles showed limitation of flexion. Dermatomyositis was diagnosed on the basis of muscle weakness and skin changes in association with a raised creatine phosphokinase activity, myopathic electromyographic changes, and typical histological muscle change. Treatment was started with prednisone $30 \mathrm{mg}$ daily $(2 \mathrm{mg} / \mathrm{kg}$ per day) (Fig. 3). Creatine phosphokinase activities decreased to normal values and there was some clinical improvement. The weakness increased slowly over the next five years, at which time creatine phosphokinase and aldolase activities were moderately raised and the rheumatoid factor became consistently positive. Later, cyclophosphamide plus prednisolone was prescribed. On the final hospital admission, at the age of 10 years 8 months, there was facial oedema, nausea, and general malaise. Her heart was enlarged (cardiopulmonary ratio $=0.6$ ) and gallop rhythm was present. Left bundle branch block was evident on the electrocardiogram. Ventricular dilatation and decreased wall motion of both ventricles were seen on the echocardiogram. Treatment was started with digoxin and

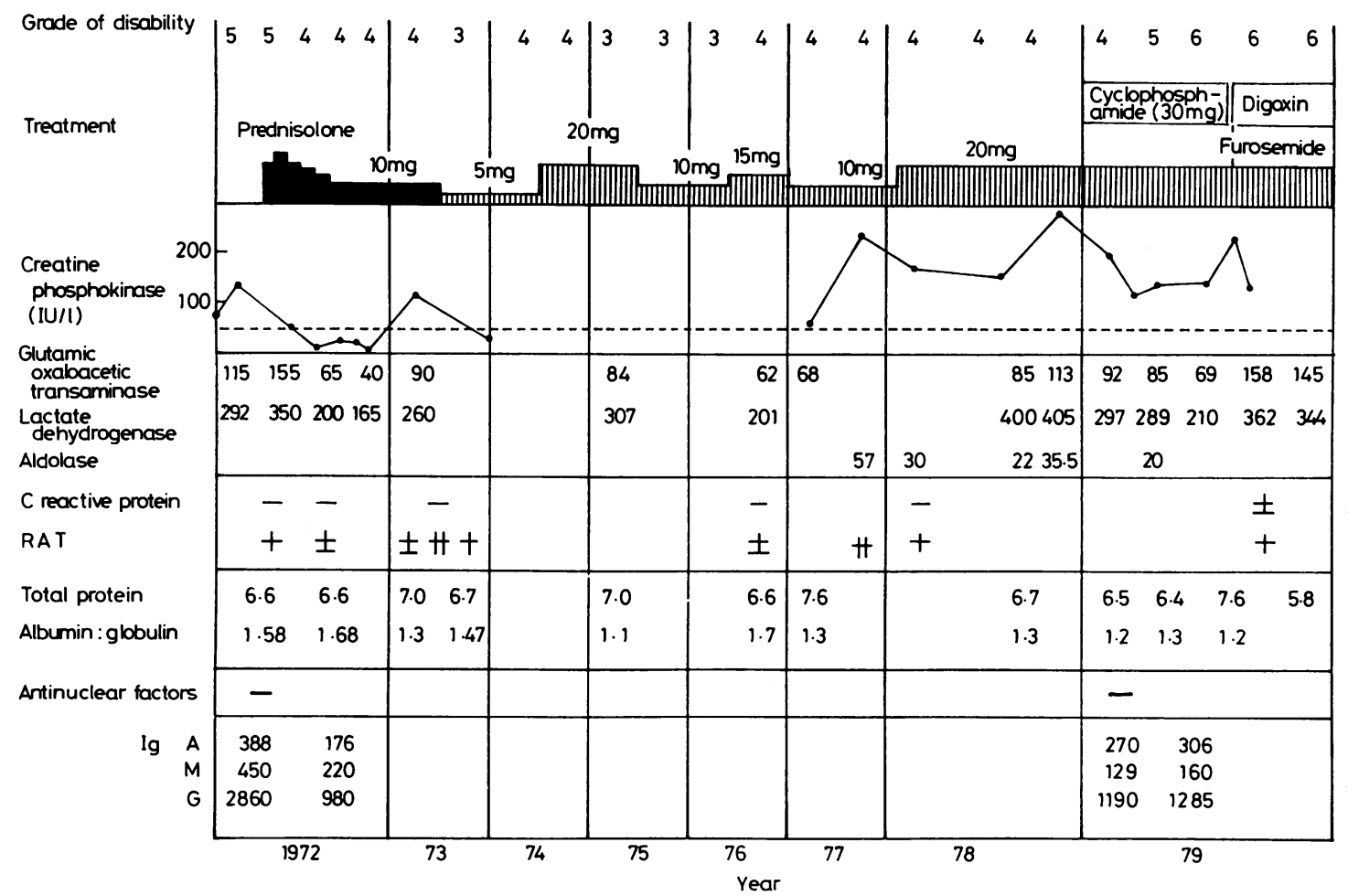

Fig. 3 Clinical course and treatment of the patient in case 13. 
furosemide. There was an initial favourable response to this regimen, but congestive cardiac failure ensued and she died at the age of 10 years 10 months.

\section{Discussion}

Polymyositis is thought to have an autoimmune basis and at present a cell mediated autoimmune reaction is widely held to be responsible for this disease. ' Treatment with corticosteroids does improve the prognosis: ${ }^{3-6} 1112$ the death rate has been reduced from around $33 \%$ to less than $10 \%$ with this management. ${ }^{3411}$ Some children, however, are refractory to steroid treatment, and for these treatment with immunosuppressive agents, or more recently, a series of plasmapheresis has been recommended. ${ }^{7-9}$ 13-16 The suggested dose of prednisolone is $2 \mathrm{mg} / \mathrm{kg} / \mathrm{day},{ }^{3}{ }^{4}$ although Dubowitz ${ }^{5}$ and Miller et $a l^{6}$ have proposed that a low dose of prednisolone is effective. ${ }^{56}$ We tried low dosage prednisolone treatment on the second admission in the patient case 2 , but he did not respond well. We then employed $2 \mathrm{mg} / \mathrm{kg} /$ day of prednisolone and were able to induce the second remission (Fig. 2).

Polymyositis in association with other connective tissue disorders is generally less likely to respond to steroid treatment than the uncomplicated disease. ${ }^{8}$ In our series, cases 13 and 10 are thought to have dermatomyositis in association with progressive systemic sclerosis and rheumatoid arthritis, respectively, and little or no improvement occurred with steroid treatment in these two. On the other hand, although case 2 has uncomplicated polymyositis, he responded only partially to steroid and immunosuppressive drugs.

There are recent reports of patients with polymyositis refractory to immunosuppressive treatment who improved with plasmapheresis, suggesting the involvement of a circulating factor, such as autoantibody or immune complexes in the production of muscle lesions. ${ }^{14}$ We examined circulating immune complexes with solid phase $\mathrm{Clq}$ binding assays in case 2 and found a slightly raised concentration once only. As cytotoxic reactivity has been experimentally shown in lymphocytes from patients with polymyositis against cultures of skeletal muscle cells, we prescribed lympho-plasmapheresis for this patient, in addition to cytotoxic immunosuppressive treatment-this led to some clinical improvement.

Cardiac involvement in adult and childhood polymyositis is an uncommon occurrence. ${ }^{17}$ Electrocardiographic evidence of pericarditis, myocarditis, and arrhythmias has been reported. ${ }^{17}$ Histological changes of fibrosis and sarcolemmic degeneration in cardiac and skeletal muscle are identical, and fibrosis of the cardiac conducting system can lead to progressive cardiac conduction defects. ${ }^{18-21}$ The patient in case 13 died of congestive heart failure 7 years from the onset of muscle weakness. The persistence of raised creatine phosphokinase activity in this patient indicated continuing progression of the underlying disease process, and the long term prognosis was unfavourable. Thus, cardiac involvement in those with polymyositis can become clinically overt if the disease persists, and the inflammatory or degenerative myocardial status may precipitate a variety of cardiac conduction defects. ${ }^{22}$ Patients with polymyositis refractory to steroid should, therefore, be treated with alternative forms of therapy.

The author is grateful to M Ohara, Kyushu University for comments on the manuscript.

\section{References}

1 Currie S. Polymyositis and related disorders. In: Walton JN, ed. Disorders of voluntary muscle. Edinburgh: Churchill Livingstone, 1981:525-68.

2 Whitaker JN, Engel WK. Vascular deposits of immunoglobulin and complement in idiopathic inflammatory myopathy. $N$ Engl $J$ Med 1972;286:333-8.

${ }^{3}$ Sullivan DB, Cassidy JT, Petty RE, Burt AB. Prognosis in childhood dermatomyositis. J Pediatr 1972;80:555-63.

${ }^{4}$ Rose AL. Childhood polymyositis: a follow-up study with special reference to treatment with corticosteroids. Am J Dis Child 1974;127:518-22.

5 Dubowitz V. Treatment of dermatomyositis in childhood. Arch Dis Child 1976;51:494-9.

6 Miller G, Heckmatt JZ, Dubowitz V. Drug treatment of juvenile dermatomyositis. Arch Dis Child 1983;58:445-50.

7 Malaviya AN, Many A, Schwartz RS. Treatment of dermatomyositis with methotrexate. Lancet 1968;ii:485-8.

${ }^{*}$ Currie S, Walton JN. Immunosuppressive therapy in polymyositis. J Neurol Neurosurg Psychiatry 1971;34:447-52.

9 Jacobs JC. Methotrexate and azathioprine treatment of childhood dermatomyositis. Pediatrics 1977;59:212-8.

${ }^{10}$ Rose AL, Walton JN. Polymyositis: a survey of 89 cases with particular reference to treatment and prognosis. Brain 1966;89:747-68.

11 Medsger TA, Robinson H, Masi AT. Factors affecting survivorship in polymyositis. Arthritis Rheum 1971;14:249-58.

12 Pearson CM. Patterns of polymyositis and their responses to treatment. Ann Intern Med 1963;59:827-38.

13 Brewer EJ, Giannini EH, Rossen RD, Patten B, Barkley E. Plasma exchange therapy of a childhood onset dermatomyositis patient. Arthritis Rheum 1980;23:509-13.

14 Dau PC. Plasmapheresis in idiopathic inflammatory myopathy: experience with 35 patients. Arch Neurol 1981;38:544-52.

15 Dau PC, Bennington JL. Plasmapheresis in childhood dermatomyositis. J Pediatr 1981;98:237-40.

16 Anderson L, Ziter FA. Plasmapheresis via central catheter in dermatomyositis: a new method for selected pediatric patients. J Pediatr 1981;98:240-1.

17 Pearson CM. Polymyositis and related disorders. In: Walton JN, ed. Disorders of voluntary muscle. Boston: Littlc Brown, 1964:501-39.

${ }^{1 \times}$ Hill DL, Barrow HS. Identical skeletal and cardiac muscle involvement in a case of fetal polymyositis. Arch Neurol 1968;19:545-51. 
${ }^{19}$ Lynch PG. Cardiac involvement in chronic polymyositis. $\mathrm{Br}$ Heart $J$ 1971:33:416-9.

20 Schaumberg HH. Nielsen SL. Yurchak PM. Heart block in polymyositis. $N$ Engl J Med 1971;284:480-1.

21 Reid JN, Murdoch R. Polymyositis and complete heart block. Br Heart J 1979;41:628-9.

22 Singsen B, Goldreyer B. Stanton R, Hanson V. Childhood polymyositis with cardiac conduction defects. Am J Dis Child 1976:130:72-4.

Correspondence to Dr M Yoshioka. Department of Paediatrics Kobe General Hospital, 4-6 Minatojima-Nakamachi. Chuo-ku. Kobe, 650 Japan.

Received 14 November 1984

\section{Commentary}

\section{VICTOR DUBOWITZ}

\section{Hammersmith Hospital, London}

The three patients with steroid resistant dermatomyositis documented by Drs Yoshioka, Okuno, and Mikawa are all highly atypical of the usual childhood dermatomyositis, as the authors themselves point out. In two of them there was evidence of other collagen disease-systemic sclerosis (case 13) and rheumatoid arthritis (case 10). The remaining patient (case 2) had what sounds like a more classic dermatomyositis, but his response to prednisolone was odd. Usually there is evidence of clinical response in the way of improved general well being and loss of the characteristic misery, and improvement in muscle function within two to four weeks of starting treatment and the creatine phosphokinase activity, if initially raised (which only occurs in about half the cases), often lags behind, remaining raised at times, even after complete clinical resolution with tailing of steroids. For this reason the clinical course is a much more reliable guide to reducing treatment than the creatine phosphokinase activity. In their case 2 , the response was completely paradoxical with a precipitous fall in the creatine phosphokinase activity without any apparent clinical improvement. Although steroid has a direct influence on creatine phosphokinase activity, it seems unlikely one could ascribe so noticeable a fall to a non-specific steroid effect. All steroids are likely to cause a steroid myopathy, but the 9 - $\alpha$-fluoro steroids even more so; so that their use of betamethasone would normally be contraindicated as a drug of choice for dermatomyositis.

With increasing build up of cytotoxic treatment in addition to continuing steroid this patient eventually showed some response. It is often assumed that lack of response to increasing treatment (or high dose initial treatment) indicates unresponsiveness, but the alternative possibility of iatrogenic steroid myopathy as a result of overtreatment is equally likely. This was well illustrated by the recent case of an 11 year old girl referred to me with refractory dermatomyositis, who had initially responded well to treatment but subsequently became resistant to increasing doses of steroids with associated increasing generalised weakness together with swallowing and respiratory difficulties. She had become bed bound for 6 weeks and was considered to be terminal. She was on $70 \mathrm{mg}$ prednisolone/day and had severe painful osteoporosis of her spine which made it almost impossible to move her. Over a period of five months we were able to gradually wean her off the steroids and get her ambulant again and as we reduced the steroids her strength steadily improved.

In the series of 29 of our personal cases reviewed by Miller $e t$ al $^{1}$ the group of patients having a low dosage of prednisolone initially had fewer relapses and less morbidity and were on steroids for a shorter period of time than those receiving higher dosage. Six of the 10 cases in the low dosage group made an uncomplicated recovery and were off treatment at least a year compared with only 1 of 18 in the second group, although the initial severity of the groups seemed comparable. Of the 12 patients in group 2 off steroids there were more severe problems with calcinosis and contractures and four were severely incapacitated.

Childhood dermatomyositis is almost uniformly responsive to steroid treatment. I think there is a good chance of remission with minimal risk of secondary complications from treatment with an initial low dosage schedule of prednisolone of 1 $\mathrm{mg} / \mathrm{kg}$ daily and a reduction as soon as there is definite sign of clinical improvement (usually within two to four weeks) either in general wellbeing and loss of misery, or in increase in muscle power. This should be done very gradually and tailored to the needs of each patient, who should be closely monitored. In a $30 \mathrm{~kg}$ child, for example, I would start on $30 \mathrm{mg}$ prednisolone/day and reduce by 5 $\mathrm{mg} /$ day at two weekly intervals till $20 \mathrm{mg} /$ day and then by $2.5 \mathrm{mg} /$ day at two weekly intervals till 10 $\mathrm{mg} / \mathrm{day}$ and then by $1 \mathrm{mg} / \mathrm{day}$ at two weekly intervals. If at any point there is a suggestion of deterioration, manifesting itself either as increasing weakness or general misery, one should go back one step in the treatment and perhaps wait an extra two weeks before reducing this, or reduce at half the rate (alternate days instead of each dav).

I reserve the use of additional drugs such as azathioprine, methotrexate, or cyclophosphamide for patients who are either incompletely responsive to steroid or who are difficult to wean off steroids. Alternate day steroid treatment is less effective and much slower for initial induction and should be 